\title{
Enlargement of a low-dimensional stochastic web
}

\author{
S.M. Soskin ${ }^{*,+,}$, I.A. Khovanov ${ }^{* *, \sharp}$, R. Mannella ${ }^{\S}$ and P.V.E. McClintock ${ }^{\ddagger}$ \\ *Institute of Semiconductor Physics, 03028 Kiev, Ukraine \\ ${ }^{\dagger}$ Abdus Salam ICTP, 34100 Trieste, Italy \\ ${ }^{* *}$ School of Engineering, University of Warwick, Coventry CV4 7AL, UK \\ ${ }^{\ddagger}$ Physics Department, Lancaster University, Lancaster LAl 4YB, UK \\ ${ }_{\S}^{\$}$ Dipartimento di Fisica, Universita di Pisa, 56127 Pisa, Italy
}

\begin{abstract}
We consider an archetypal example of a low-dimensional stochastic web, arising in a 1D oscillator driven by a plane wave of a frequency equal or close to a multiple of the oscillator's natural frequency. We show that the web can be greatly enlarged by the introduction of a slow, very weak, modulation of the wave angle. Generalizations are discussed. An application to electron transport in a nanometre-scale semiconductor superlattice in electric and magnetic fields is suggested.
\end{abstract}

Keywords: stochastic webs, separatrix chaotic layer, chaotic transport, semiconductor superlattice PACS: PACS numbers: 05.45.-a,72.20.Ht

\section{INTRODUCTION}

In weakly perturbed integrable Hamiltonian systems, small areas of the phase space near resonances are chaotic [1]. The stochastic web concept dates back to the early 1960s when Arnold showed [2] that, in non-degenerate Hamiltonian systems of dimension exceeding 2, resonance lines necessarily intersect, forming a web of infinite-size in the Poincaré section. It provides in turn for a slow chaotic (sometimes called "stochastic") diffusion over infinite distances in the relevant dynamical variables.

It was discovered at the end of 1980s [3-5] that, in degenerate or nearly degenerate systems, stochastic webs can arise even for dimension 3/2. An archetypal example of such a low-dimensional stochastic web arises when a 1D harmonic oscillator is perturbed by a weak traveling wave whose frequency coincides with a multiple of the natural frequency of the oscillator $[1,4,6]$. Perturbation plays a dual role: on the one hand, it gives rise to a slow dynamics characterized by an auxiliary Hamiltonian that possesses an infinite web-like separatrix; on the other hand, the perturbation destroys this selfgenerated separatrix, replacing it with a thin chaotic layer. Such a low-dimensional stochastic web may be relevant to a variety of physical systems and plays an important role in the corresponding transport phenomena: see $[1,4,6]$ for reviews on relevant classical systems. There are also quantum systems the dynamics of whose transport reduces to that of the classical model described above. One example is a nanometre-scale semiconductor superlattice with an applied voltage and magnetic field [7, 8]. Moreover, there is evidence that, if a classical system possesses a stochastic web, then transport in the quantum analogue of that system is much stronger than where the classical system does not possesses a web $[9,10]$, a finding that may be relevant e.g. to the transport of ultra-cold atoms in optical lattices [9].

One might assume that, like the Arnold web, the low-dimensional stochastic web 
described above should be infinite, so that it would provide for transport between the centre of the web and states situated arbitrarily distant in coordinate and momentum. However this is not the case. Provided that the perturbation is not exponentially strong, the real web is confined to the region within just a few inner loops of the infinite web-like resonant separatrix (cf. $[1,4,6]$ ). The reason is as follows. The resonant Hamiltonian possesses a single, infinite, web-like separatrix only in the first-order approximation of the averaging method [11]; with account taken of the next-order approximations, the separatrix splits into many separate complex loops successively embedded within each other. Non-resonant terms of the perturbation dress the separatrices by exponentially narrow chaotic layers. If the perturbation is not small, the chaotic layers manage to connect neighbouring separatrix loops situated close to the centre. However, the width of the chaotic layer decreases exponentially fast with increasing distance from the centre $[1,4,6]$. As a result, coalescence of the chaotic layers associated with adjacent loops only occurs among the few loops closest to the centre.

If the resonance between the perturbation and the oscillator is inexact, or if the oscillator is nonlinear, the separation of neighbouring loops is typically much larger: it is already apparent in the first-order approximation of the averaging method $[1,5,6]$. So, the number of the loops connected to the centre by chaotic transport is even smaller $[1,5,6]$ than in the case of an exact resonance.

How can one modify the perturbation in order that the transport should become unlimited or, at least, significantly extended? One possible answer was obtained at the very beginning of the studies of low-dimensional webs [3]: if the perturbation consists of short kicks that are periodic in space and time, and if the frequency of the kicks is equal to a multiple of the natural frequency, then a so called uniform web is formed covering the whole phase space. Such a perturbation seldom applies in practice, however, and even where it does the chaotic transport is still exponentially slow [1,6].

Is it possible to obtain a web of form similar to the original one [4] but substantially extended in the phase space? Our present work leads to a positive answer based on the following simple idea. The chaotic layer in the web is exponentially narrow because the frequency of the non-resonant perturbation of the resonant Hamiltonian is necessarily much higher than the frequency of small eigenoscillations in the cell of the web-like separatrix [1,3-6]. So, we need to modify the perturbation in such a way that the resonant Hamiltonian does not change while its perturbation contains, in addition to the conventional terms, a low-frequency one. One may do this by modulating the wave angle at a low frequency or by adding one more wave of frequency shifted slightly from the original one. The latter option will be considered elsewhere together with a generalization for the uniform web (leading to a huge enhancement of the chaotic transport through it). Our present work concentrates on the first option since it may have immediate applications to nanometre-scale semiconductor superlattices in electric and magnetic fields $[7,8]$. 


\section{SLOW MODULATION OF THE WAVE ANGLE}

Fig. 1 demonstrates the efficiency of our method. We integrate the equation

$$
\ddot{q}+q=0.1 \sin [15 q-4 t-h \sin (0.02 t)],
$$

first for $h=0$ (i.e. for the conventional case with parameters as in $[1,4,6]$ ), and secondly for $h=0.1$. Although the modulation in the latter case is weak (its amplitude is small compared to the $2 \pi$ period of the wave angle), the resultant increase in size of the web in coordinate and momentum is large: a factor of about $6 \times$.

To account for these results we develop the analytic theory, generalized for the offresonant case $[1,5,6]$, using a general method developed recently in [12]. It is anticipated that the method can also be generalized for uniform webs $[1,3,6]$, leading to an exponentially strong enhancement of chaotic transport through them.
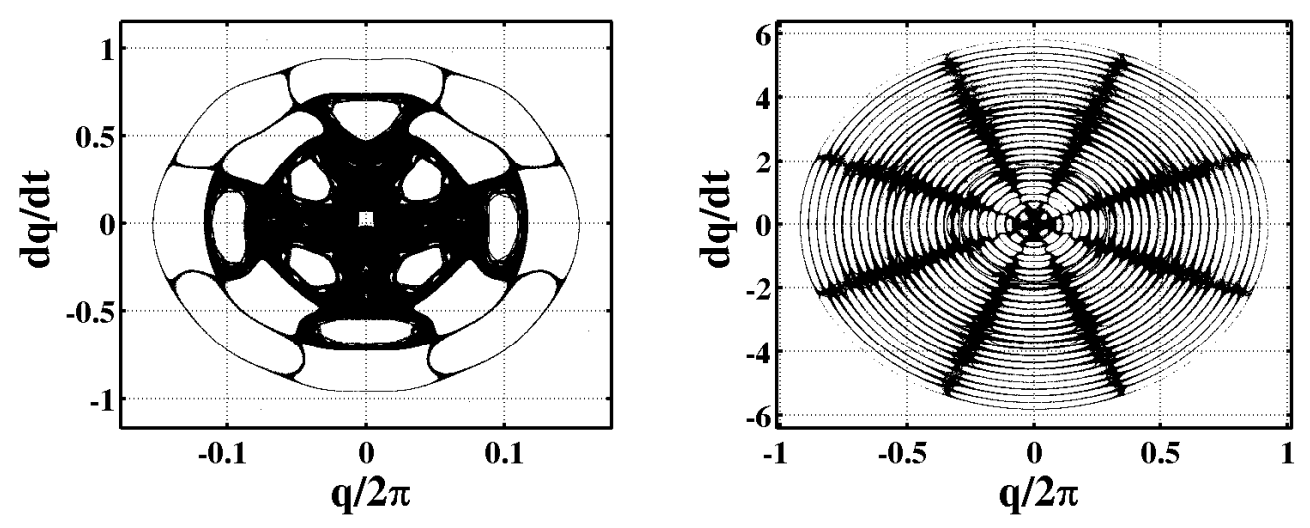

FIGURE 1. The Poincaré section for a trajectory of the system (1) with initial state $q=0.1, \dot{q}=0$ (at instants $t_{n}=n T$ where $T \equiv 2 \pi / 0.02$ is the period of the modulation and $n=1,2,3, \ldots 600000$ ) for $h=0$ (left panel) and $h=0.1$ (right panel). A symplectic integration scheme of the fourth order is used, with an integration step $t_{\text {int }}=\frac{2 \pi}{40000} \approx 1.57 \times 10^{-4}$, so that the inaccuracy at each step is of the order of $t_{\text {int }}^{5} \approx \times 10^{-19}$. The left panel corresponds to the conventional case considered in $[1,4,6]$. The right panel demonstrates that the modulation, although weak, greatly enlarges the web sizes (note the different axes scales), thereby greatly enhancing the chaotic transport.

\section{APPLICATION TO SEMICONDUCTOR SUPERLATTICES}

We now consider application to quantum electron transport in nanometre-scale 1D semiconductor superlattices (SLs) subject to a constant electric field along the SL axis and to a constant magnetic field $[7,8]$. The spatial periodicity gives rise to minibands for the electrons. In the tight-binding approximation, the electron's energy $E$ as a function of its momentum $\vec{p}$ in the lowest miniband is given by

$$
E(\vec{p})=\frac{\Delta\left[1-\cos \left(p_{x} d / \hbar\right)\right]}{2}+\frac{p_{y}^{2}+p_{z}^{2}}{2 m^{*}},
$$

where $x$ is the direction along the SL axis, $\Delta$ is the miniband width, $d$ is the SL period, $m^{*}$ is the electron effective mass for the motion in the transversal (i.e. $y-z$ ) direction. 
Thus, the quasi-classical motion of an electron of charge $e$ in an electric field $\vec{F}$ and a magnetic field $\vec{B}$ is described by:

$$
\frac{\mathrm{d} \vec{p}}{\mathrm{~d} t}=-e\left\{\vec{F}+\left[\nabla_{\vec{p}} E(\vec{p}) \times \vec{B}\right]\right\}
$$

It was shown in [7] that, for constant electric field along the SL axis $\vec{F}=\left(-F_{0}, 0,0\right)$ and constant magnetic field with a given angle $\theta$ to the axis $\vec{B}=(B \cos (\theta), 0, B \sin (\theta))$, the dynamics of the $z$-component of momentum $p_{z}$ reduces to the equation of motion of an auxiliary harmonic oscillator in a plane wave. At certain values of the parameters, the ratio of the wave and oscillator frequencies takes integer values (as in Eq. (1) with $h=0$ ) giving rise to the onset of the stochastic web. This leads in turn to a delocalization of the electron in the $x$-direction and, consequently, to an increase of the dc-conductivity along the SL axis. The experiment [8] appears to provide evidence in favor of this exciting hypothesis.

At the same time, the finite size of the web and, yet more so, the exponentially fast decrease of the transport rate as the distance from the centre of the web increases, seem to place strong limitations on the use of the effect. We now suggest a simple and efficient way to overcome these limitations: if we add to the original (constant) electric field $F_{0}$ a small time-periodic (ac) component $F_{\mathrm{ac}} \cos \left(\Omega_{\mathrm{ac}} t\right)$, then the wave angle in the equation of motion of $p_{z}$ is modulated by the following term (cf. Eq. (1)):

$$
h \cos (\Omega t) \equiv \frac{F_{\mathrm{ac}}}{F_{0}} \frac{\Omega_{0}}{\Omega_{\mathrm{ac}}} \cos \left(\frac{\Omega_{\mathrm{ac}}}{\Omega_{0}} t\right), \quad \Omega_{0} \equiv \frac{e F_{0} d}{\hbar} .
$$

This allows us to increase drastically the size of the web and the rate of chaotic transport through it. For example, for the case shown in Fig. 1, where we have an increase of the web size by the factor of $6 \times$, it is sufficient to add an ac component of electric field of frequency $0.02 \times \Omega_{0}$ and amplitude $F_{\mathrm{ac}}=0.1 \times 0.02 \times F_{0}$, i.e. an amplitude that is $500 \times$ smaller than the original constant field $F_{0}$ !

\section{REFERENCES}

1. G.M. Zaslavsky, Physics of Chaos in Hamiltonian systems, Imperial Colledge Press, London, 2007.

2. V.I. Arnold, Dokl. Acad. Nauk SSSR 156, 9 (1964).

3. G.M. Zaslavsky et al., Sov. Phys. JETP 64, 294 (1986); A.A. Chernikov et al., Nature 326, 559 (1987).

4. A.A. Chernikov et al., Phys. Lett. A 122, 39 (1987).

5. A.A. Chernikov et al., Phys. Lett. A 129, 377 (1988).

6. G.M. Zaslavsky, R.D. Sagdeev, D.A. Usikov and A.A. Chernikov, Weak Chaos and Quasi-Regular Patterns, Cambridge University Press, Cambridge, 1991.

7. T.M. Fromhold et al., Phys. Rev. Lett. 87, 046803 (2001).

8. T.M. Fromhold et al., Nature 428, 726 (2004).

9. W.K. Hensinger et al., Nature 412, 52 (2001); D.A. Steck, W.H. Oskay, M.G. Raizen, Science 293, 274 (2001); R.G. Scott et al., Phys. Rev. A 66, 023407 (2002).

10. A.R.R. Carvalho, A. Buchleitner, Phys. Rev. Lett. 93, 204101 (2004).

11. N.N. Bogolyubov, Yu.A. Mitropolsky, Asymptotic Methods in the Theory of Nonlinear Oscillators, Gordon and Breach, New York, 1961.

12. S.M. Soskin, R. Mannella, and O.M. Yevtushenko, Phys. Rev. E 77, 036221 (2008). 